Article

\title{
Tune That Beer! Listening for the Pitch of Beer
}

\author{
Felipe Reinoso Carvalho ${ }^{1,2, *}$, Qian (Janice) Wang ${ }^{3}$, Brecht de Causmaecker ${ }^{4}$, Kris Steenhaut ${ }^{1}$, \\ Raymond van Ee ${ }^{2,5,6}$ and Charles Spence ${ }^{3}$ \\ 1 Department of Electronics and Informatics (ETRO), Vrije Universiteit Brussel, 1050 Brussels, Belgium; \\ ksteenha@etrovub.be \\ 2 Brain \& Cognition, Laboratory of Experimental Psychology, KU Leuven, 3000 Leuven, Belgium; \\ raymond.vanee@kuleuven.be \\ 3 Crossmodal Research Laboratory, Department of Experimental Psychology, Oxford University, \\ Oxford OX1 3UD, UK; qian.wang@psy.ox.ac.uk (Q.W.); charles.spence@psy.ox.ac.uk (C.S.) \\ 4 Laboratory of Enzyme, Fermentation and Brewing Technology (EFBT), KU Leuven Technology Campus, \\ 9000 Gent, Belgium; brecht.decausmaecker@kuleuven.be \\ 5 Department of Brain, Body \& Behavior, Philips Research Laboratories, 5656 AE Eindhoven, The Netherlands \\ 6 Department of Biophysics, Donders Institute, Radboud University, 6500 GL Nijmegen, The Netherlands \\ * Correspondence: freinoso@vub.ac.be; Tel.: +32(0)-2-559-1528
}

Academic Editor: Miranda Mirosa

Received: 21 September 2016; Accepted: 11 November 2016; Published: 17 November 2016

\begin{abstract}
We report two experiments designed to assess the key sensory drivers underlying people's association of a specific auditory pitch with Belgian beer. In particular, we assessed if people would rely mostly on the differences between beers in terms of their relative alcohol strength, or on the contrast between the most salient taste attributes of the different beers. In Experiment 1, the participants rated three bitter beers (differing in alcohol content), using a narrow range of pitch choices $(50-500 \mathrm{~Hz})$. The results revealed that the beers were all rated around the same pitch (Mean $=232 \mathrm{~Hz}$, $\mathrm{SD}=136 \mathrm{~Hz}$ ). In Experiment 2, a wider range of pitch choices (50-1500 Hz), along with the addition of a much sweeter beer, revealed that people mostly tend to match beers with bitter-range profiles at significantly lower pitch ranges when compared to the average pitch of a much sweeter beer. These results therefore demonstrate that clear differences in taste attributes lead to distinctly different matches in terms of pitch. Having demonstrated the robustness of the basic crossmodal matching, future research should aim to uncover the basis for such matches and better understand the perceptual effects of matching/non-matching tones on the multisensory drinking experience.
\end{abstract}

Keywords: beer; flavor; taste; sound; alcohol content; pitch; crossmodal correspondences

\section{Introduction}

The soundscapes in the places where we eat and drink-such as restaurants, bars, and airplanes - exert a significant influence over our perception of the taste and flavor of food and drink ([1]; see [2,3] for reviews). Methods and techniques are undergoing increasing innovation in order to further evaluate how a certain sound can alter the perception of different food attributes, such as sweetness (e.g., [4-10]; see [11] for a review).

That there might be crossmodal links between sounds (e.g., pure tones) and basic tastes (e.g., sweet, bitter, sour, and salty) is by no means obvious. However, a number of recent studies have highlighted the robustness of such associations, enriching the discussion on why it is that people associate specific sounds with particular tastes, aromas, textures, and flavors (see [11] for a review). Beyond the basic matching of seemingly-unrelated stimuli across the senses, the latest research has also demonstrated how sound can influence the multisensory tasting experience (see $[5,11]$ for overviews). Besides researching the potential influences of the sound of food being consumed, researchers have also 
examined how sounds that are unrelated to food and drink can nevertheless exert a significant influence over multisensory tasting experiences (see [12,13] for reviews). For instance, Reinoso Carvalho and his colleagues [7] conducted an experiment in which the customers in a chocolate shop rated a chocolate before eating it, versus eating it while listening to different soundscapes (including a song). The results revealed that these participants were willing to pay significantly more for the song-chocolate experience, as compared to their ratings before having such a multisensory experience.

A pioneering study from the late 1960s has become a general source of inspiration for many of those working in this area of research: Holt-Hansen [14] reported that participants made consistent associations between audible frequency ranges (pitch) and two beers. Here, the participants were presented with iterative frequency sweeps on a tone generator, to select the pitch that they thought best matched the flavor of each of the two beers. The participants matched Carlsberg Elephant Beer, a beer with higher alcohol content, to a significantly higher range of frequencies (640-670 Hz), when compared to regular Carlsberg lager (510-520 Hz). Some years later, Rudmin and Cappelli [15] reported a partial replication of the aforementioned experiment with 10 participants. In the latter study, though, beer was not the only stimulus that the participants were given to match. Here, a range of five foods/drinks was presented, comprising non-alcoholic beer, Carlsberg lager beer, grapefruit juice, a hard candy, and a dill pickle. When comparing the studies of Holt-Hansen [14] to that of Rudmin and Cappelli [15], the same beer (Carlsberg lager) that was matched around a pitch of $510-520 \mathrm{~Hz}$ in [14] was matched to a pitch of $600 \mathrm{~Hz}$ in [15].

More recently, various studies have highlighted consistent and robust crossmodal associations between basic taste stimuli and auditory tones. Generally speaking, sweet and sour tastes tend to be matched with a higher pitch range than either bitter or salty tastes $[16,17]$.

The research reported here analyzed whether participants would consistently associate different flavors of beer with different pitches. We reassess the hypothesis raised by the studies of Holt-Hansen and Rudmin and Cappelli $[4,14,15]$, but now focusing on better disentangling the main flavor attributes that may be behind these beer-pitch associations. Four different Belgian beers were used in this 'tuning' study. The study was subdivided in two experiments. Each experiment consisted of 'tuning' three different beers. In the first experiment, the beers were chosen to provide a range of alcohol contents. Here, we were motivated by the fact that in Holt-Hansen's [14] results, the participants matched the beer that just so happened to have the higher alcohol content to a significantly higher pitch. In the second experiment, however, the beers were chosen based on their differences in levels of sweetness and bitterness, as it has been previously argued that sweet tastes tend to be matched with a higher pitch range than bitter tastes [16,17].

In other words, we wondered if people would match different pitches based on high differences in alcohol content, as Holt-Hansen discussed in his studies, or, perhaps, if they would rely more on a clear difference in whatever the most salient taste differences between the beers happen to be as the main reference for these multisensory matching tasks.

\section{Materials and Methods}

\subsection{Participants}

Two experiments were conducted for this study. The first on the 8 December 2015, at the Vrije Universiteit Brussel-VUB (Experiment 1), and the second, on the 27 August 2016, at Savoie's Cantina, in Brussels (Experiment 2). The Social and Societal Ethics Committee at KU Leuven (SMEC) approved this protocol (Registered as G-2015 07 282). In both experiments, general visitors or customers were invited to take part in a short experiment. They were informed that they would be given complimentary beer to taste while listening to sounds and answering a survey. A total of 75 participants took part in the two experiments ( $29 \%$ females, $71 \%$ males, mean (M) age of 24.8 years, standard deviation (SD) of 7.0). All of the participants were at least, 16 years of age (the minimum legal age to drink alcohol in Belgium), and gave their informed consent prior to taking part in the study. None of the 
participants reported having a cold or any other impairment of their senses of smell, taste, or hearing at the time of the study. In general, the participants were Belgian residents, especially from Brussels and its surroundings. In particular, they reported having a mid-high knowledge of the types and brands of Belgian beer $(\mathrm{M}=3.17, \mathrm{SD}=1.21)$ — these ratings were based on five-point scales, with 1 being 'Not at all', and 5 'Very much'. They also reported as drinking beer often $(\mathrm{M}=4.70, \mathrm{SD}=1.71)$-these ratings based on seven-point scales, with 1 being 'Never', and 7 'Very often'. Finally, when asked about their music 'expertise', most people reported as being intermediate (corresponding to less than 10 years of music-playing experience).

\subsection{Stimuli}

Three Belgian beers were used in Experiment 1. Taras Boulba (Beer A) is a dry Pale Ale beer labeled as $4.5 \%$ in alcohol strength and 43.1 IBU (IBU is the standardized scale for bitterness in beers). Zinnebir (Beer B) is a golden blond Pale Ale beer labeled as 6\% in alcohol strength. Jambe de Bois (Beer C) is a full-bodied ${ }^{1}$ blond and copper-colored Tripel beer. Beer $C$ is the strongest of the three (labeled as $8 \%$ in alcohol strength), and has 47.7 IBU. Beer B is the highest within the IBU scale (51.5), although its difference with Beer $C$ may be insignificant. Overall, these three beers are within the bitter beer scope (all beers with an IBU over 25 are usually considered as high in bitterness). In particular, their differences can be described in terms of their alcohol content (with Beer A being the lightest, and Beer $C$ the strongest), and, to a lesser degree, by their bitterness (with Beer A having the lowest IBU). Beer A may also be considered as the least sweet due to its dryness, and Beer $\mathrm{C}$ the one with more body, typical to a Tripel ${ }^{2}$ style beer. Finally, the $\mathrm{pH}$ and acidity of these beers are quite similar, which in principle does not allow them to be chemically ranked in terms of sourness. A fourth beer was used in Experiment 2, along with Beers A and C (Belle-Vue Traditional Kriek Lambic-Fruit, namely Beer D). This is a very fruity and sweet beer, where fruits are added after the spontaneous fermentation (in this case, of cherries). It is labeled as having $5.1 \%$ of alcohol content. Kriek beers usually have an IBU of between 15 and 21 (information retrieved from [20]), which makes this beer much less bitter than the other three beers.

All of the beers were served in $5 \mathrm{cl}$ samples in opaque black plastic cups that obscured the color of the drinks being tasted.

Auditory stimuli: The auditory stimuli used in this study consisted of a digital version of an adjustable pitch tone generator (also known as a frequency sweeper). Using the Online Tone Generator (retrieved from http://www.szynalski.com/tone-generator/) the participants had the opportunity to choose different tone frequencies while hearing them in real-time, in order to find the pitch that was most suitable for the flavor of each beer being tasted. Figure 1 shows an image of the corresponding graphic interface.

In order to define the available pitch range for participants to choose from, a pilot study was conducted. In this study, participants $(n=75)$ chose a pitch that matched the flavor of three bitter Belgian beers (beers A, B, and C) compared to a sweet carbonated beverage (namely 'sweet Cola'). The initial range for the pilot was between $50-1500 \mathrm{~Hz}$, and this choice was primarily based on three factors. First, the fact that the headphones started to distort the sounds below $50 \mathrm{~Hz}$. Second, Holt-Hansen [14] highlighted beer-pitch matches under $1000 \mathrm{~Hz}$. Third, the formulas of these beers are more bitter, when compared to lager beers, which is the beer type that was used in Holt-Hansen's [14] research. Hence, in this pilot, we would also expect that participants would be rating even more towards the lower part of the frequency range $[16,17]$.

1 The body of a beer is most commonly associated to its density. However, stronger beers are usually perceived as having more body as well. The mouthfeel of carbonation may also influence a beer's perceived body $[18,19]$.

2 Tripel beers use up to three times more malt than standard beers. They usually have dense-creamy heads and are beers with a full body and a higher alcohol content. 


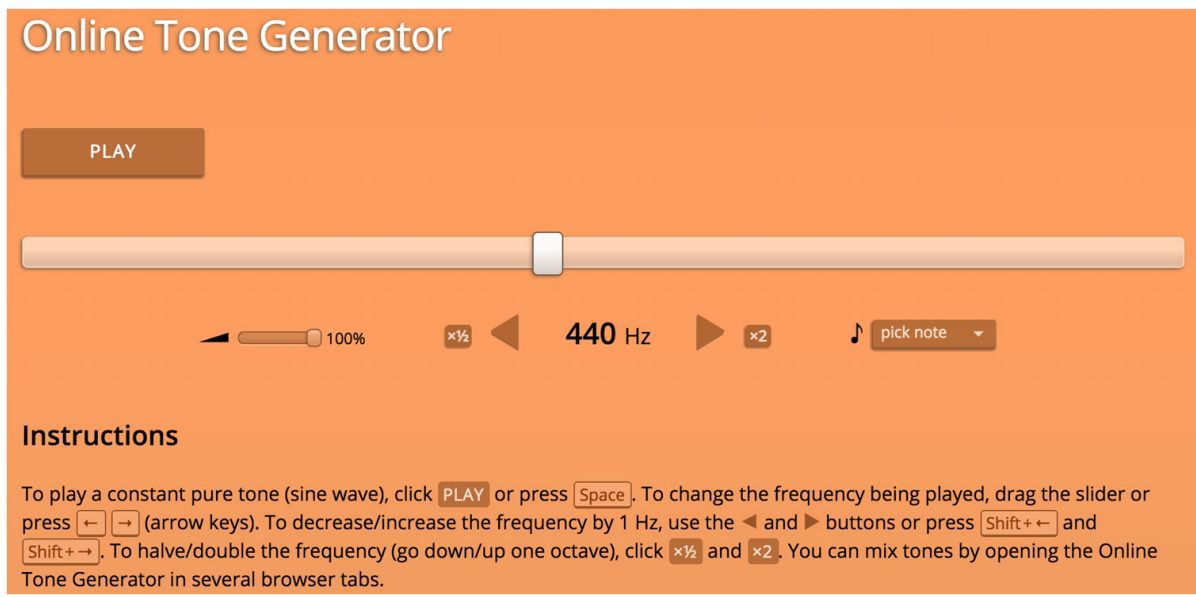

Figure 1. Graphic interface of the tone generator used in these experiments. When the interface first loads, the initial position of the controller is set at $440 \mathrm{~Hz}$. The participants could then click and drag the mouse and sweep. The pitch of the tone increased as the controller was moved to the right, and decreased as the controller was moved leftward. If preferred, the participants also had the chance to choose specific notes by selecting the 'pick note' option. A fine-volume control (left) and extra instructions (bottom) were also available.

The results of the pilot study revealed that the majority of the bitter beer ratings were within a much narrower range than the available choices (see Figure 2). For instance, the boxplots show that the medians of Beers A, B, and C are situated around $200 \mathrm{~Hz}$, whereas the median of the cola beverage seems to be around $400 \mathrm{~Hz}$ (twice as high). These median values also show us that the differences in the three bitter beer rates seem to be minimally influenced by their differences in alcohol content. The ratings of Beer $\mathrm{C}$ would seem, though, to be more dispersed than the other beers. In fact, the corresponding boxplot shows approximately twice the number of outliers. This probably has to do with its higher alcohol content, when compared to the other two beers (as in [14]). Considering the results of this pilot (along with the revised literature), it was decided to define the pitch ranges for Experiment 1 as between 50 and $500 \mathrm{~Hz}$, and, for Experiment 2, between 50 and $1500 \mathrm{~Hz}$ (here, a sweeter beer was being added as a taste stimulus).

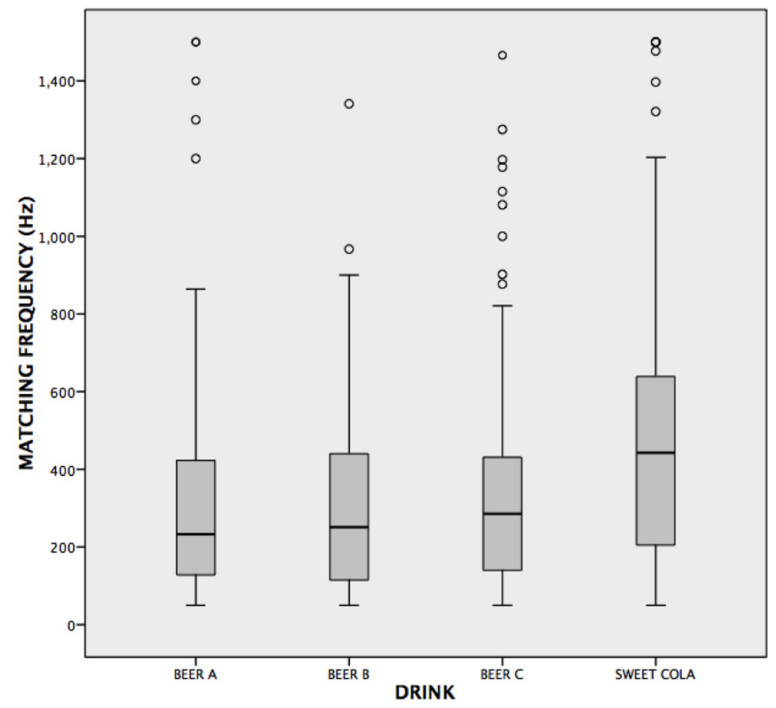

Figure 2. Participants' pitch choices during the pilot (presented as boxplots), where it is possible to appreciate that most of the results of the beer ratings are below $500 \mathrm{~Hz}$ (see [21] for an overview of how to read boxplots and their use in statistical analyses). 


\subsection{Experimental Design}

The objective of Experiment 1 was to assess whether the participants would be able to match each of the three beers with different pitch ranges (in this case, Beers A, B, and C). This experiment was conducted at the Stoa (an annexed room of the VUB's restaurant), with a total of 55 participants. Considering the results of the pilot, we chose to give the participants a reduced range of frequencies to choose from $(50-500 \mathrm{~Hz})$.

In order to test whether participants were able to place beers with very different flavors within different pitch ranges, another beer was included in Experiment 2. Here, one of the three beers from the previous experimental set-up was replaced with a beer having a very different-and sweeter-taste (Beer D). Since beers A and C have the biggest chemical differences, it was decided to keep them along with the new Beer D. With the addition of a sweeter beer, and, again, considering the results of the pilot, here, the participants were given a wider range of frequencies to choose from $(50-1500 \mathrm{~Hz})$. Twenty participants took part in this study, developed at the Savoie's Cantina.

\subsection{Experimental Procedure}

During both experiments, it was possible to have a well-controlled environment. The experiments were performed using computers placed on tables. The participants sat together, although fairly well separated, in order to prevent them from interacting. The intensity of the artificial lighting in the experimental area was dimmed to a minimum, in order to provide a more 'intimate' atmosphere.

Each participant was seated in front of a computer screen with a pair of headphones, a computer mouse, and a keyboard to complete the survey. Each computer was set to a listening level of approximately $70(+/-5 \mathrm{dBA})$. The pitch task was presented over SONY MDRZX310 headphones. The participants were not able to hear the sounds from the others participants' headphones. The survey consisted of an electronic form containing three main steps, with five- or seven-point Likert scales, multiple choice questions, and YES/NO questions. In the first step of the survey, the participants were instructed to read and accept the conditions of the informed consent before entering their demographic details. In a second step, they responded to a pre-questionnaire, in which they outlined their basic knowledge of Belgian beers. The third step of the experiment consisted of matching the flavor of the three different beers available with pure tones, while manipulating the sweep until they reached the 'ideal pitch match'. This matching process was made while participants tasted the beer, that is, while having the beer in their mouths. Once the match had been made, the participants entered the corresponding frequency value into a text box. Each participant was provided with three labeled cups, and the pitch-matching process was repeated three times, once for each beer. Separate cups with tap water for mouth rinsing were available as well. After each pitch-beer matching exercise, the participants answered a number of questions, including taste/flavor ratings and hedonic evaluations.

In order to shed more light on how this tuning process took place, in Experiment 2, a second round of questions was added. Here, the participants were asked to rate basic taste attributes of the beers (sweetness and bitterness), alcohol strength, as well as how much they liked each of the beers. All of these ratings were based on 7-point scales, with 1 representing 'Not at all' and 7 representing 'Very much' (i.e., 'Not at all sweet', compared to 'Very sweet', and so on). All the data from Experiments 1 and 2 were analyzed using SPSS.

As a final qualitative evaluation, in Experiment 2, the participants were also asked to explain (in their own words, using a comment box), how they had gone about making their beer-tuning choices.

The order of presentation of the beers was fully randomized. Hence, the participants were advised to follow the survey instructions carefully, in order to drink the corresponding beer at all times. At least one supervisor was present during the entire process to provide extra guidance, coordination, and support, in addition to the written guidelines concerning the experiment. Upon finishing the study, the participants were instructed to leave the room without discussing any details with the next group of participants. 


\section{Results}

\subsection{Experiment 1}

The pitch chosen for Beer A was $\mathrm{M}=214 \mathrm{~Hz}, \mathrm{SD}=126 \mathrm{~Hz}$. For Beer B, the chosen pitch was $\mathrm{M}=232 \mathrm{~Hz}, \mathrm{SD}=142 \mathrm{~Hz}$, while for Beer C it was $\mathrm{M}=250 \mathrm{~Hz}, \mathrm{SD}=141 \mathrm{~Hz}$ (see Figure 3). A repeated measures analysis (RM-ANOVA) was further conducted with the aforementioned pitch results as a covariate. The results revealed no significant differences between the chosen frequencies for the three beers $(F(2,106)=0.99, p=0.37$, partial eta squared $=0.02)$.

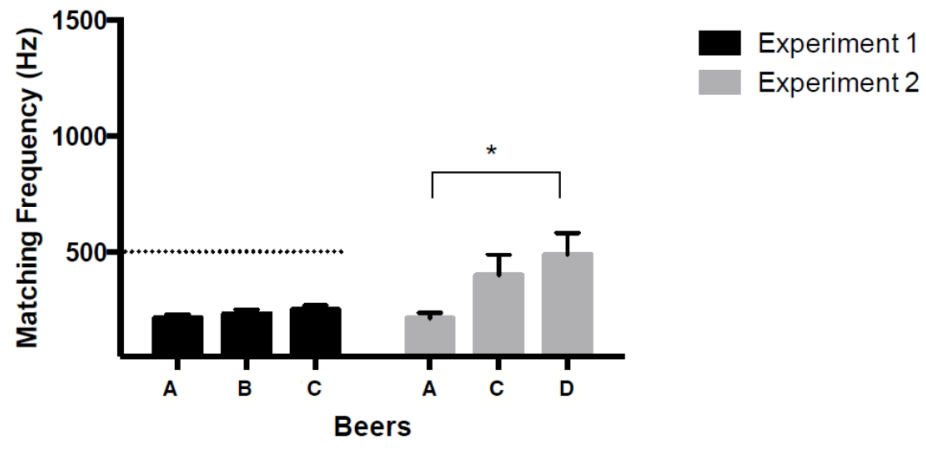

Figure 3. Means and SE of the tuning ratings of the participants in Experiments 1 and 2. The asterisk indicates a significant difference between ratings. Note that the dotted line refers to the upper limit of the pitch range available to choose from in Experiment $1(500 \mathrm{~Hz})$.

\subsection{Experiment 2}

The tuning results for Beer A were $\mathrm{M}=213 \mathrm{~Hz}, \mathrm{SD}=111 \mathrm{~Hz}$; for Beer C, M= $399 \mathrm{~Hz}, \mathrm{SD}=399 \mathrm{~Hz}$; and for Beer D, $M=487 \mathrm{~Hz} \mathrm{SD}=418 \mathrm{~Hz}$. When submitting these frequencies to a RM-ANOVA, an overall significant difference was obtained between the comparisons of the three rates $(F(2,18)=4.98$, $p=0.019$, partial eta squared $=0.36)$. Pairwise comparisons-Bonferroni corrected-revealed that the participants chose a significantly higher pitch for Beer D when compared to Beer A $(p=0.019)$. Moreover, a weak trend suggests that the participants may have chosen a higher pitch for Beer D when compared to Beer C ( $p=0.136)$. In summary, in Experiment 2, Beer D was matched approximately to a pitch that was twice as high as that for Beer A. Figure 3 shows the means and SE of all the 'tuning' ratings, on Experiments 1 and 2.

The frequencies of the second round of questions, which were specifically included as part of Experiment 2, were also analyzed. Here, individual ANOVAs (one for each question) revealed significant differences for sweetness $(F(2,22)=19.35, p<0.005$, partial eta squared $=0.638)$, bitterness $(F(2,22)=7.37, p=0.016$, partial eta squared $=0.401)$, and strength ratings $(F(2,22)=5.69, p=0.040$, partial eta squared $=0.401)$. No differences were reported for liking ratings $(F(2,22)=1.09, p=1$, partial eta squared $=0.09)$. In particular, Beer $\mathrm{D}$ was rated as sweeter $\left(p_{\mathrm{AD}}=0.002 ; p_{\mathrm{CD}}=0.002\right)$, less bitter $\left(p_{\mathrm{AD}}=0.052 ; p_{\mathrm{CD}}=0.032\right)$, and less strong $\left(p_{\mathrm{AD}}=0.006 ; p_{\mathrm{CD}}=0.041\right)$ than beers A and C. Figure 4 shows these comparisons, with their significance levels.

During the qualitative evaluation ${ }^{3}$ included in Experiment 2, the most common answer referred to the idea that bitterness was associated with a lower pitch, whereas sweetness/fruitiness were associated with a higher pitch (eight out of 20 participants). The second most common answer concerned the strength versus lightness of the beers as their references for rating $(n=4)$. The third most common answer referred to the chosen pitch as based on the pleasantness induced by the sound while being played $(n=3)$.

3 Here, the participants explained, in their own words, how their beer-tuning choices were made. 


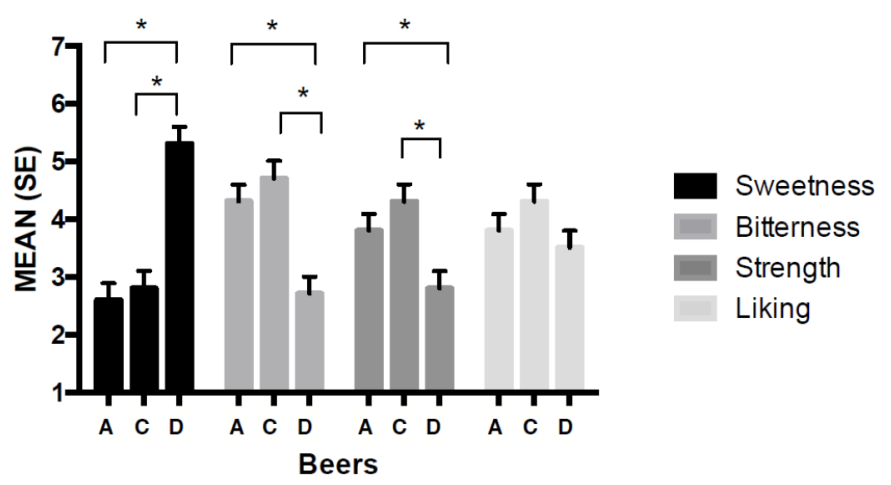

Figure 4. Means and standard error (SE) of sweetness, bitterness, strength, and liking ratings for the three beers in Experiment 2 (asterisks show a significant difference between ratings). Here, it can be seen that all the significant ratings are related to Beer $\mathrm{D}$, the sweetest one. Moreover, most significant differences reported here follow the real differences in these beers' formulas, expect for the fact that Beer A was rated as stronger than Beer D (Beer A has 4.5\% alcohol strength, and Beer D 5.1\%).

\subsection{Summary of Results}

Considering the results of both experiments, there was only one important significant difference reported between the beer-pitch matches, and this was between beers A and D, in Experiment 2 (see Figure 3). In other words, the pitch-matching task that was designed to focus on the differences in the beers' alcohol strength gave rise to inconclusive results (Experiment 1). On the other hand, the pitch-matching tasks that mostly relied on the differences in taste attributes showed significant differences (Experiment 2).

\section{Discussion}

In these two experiments, we first measured people's ability to choose the pitch for three Belgian bitter beers with considerable differences in alcohol strength (Experiment 1). Having obtained a similar pitch range for all three of the beers, participants made significantly different pitch choices between beers with very different taste attributes (Experiment 2). The results of Experiment 2, with the inclusion of a sweeter beer (Beer D), supported the interpretation that the participants were primarily rating the bitter beers towards the lower end of the pitch range (Beers A and C). By contrast, Beer D was systematically matched to a higher pitch (when compared to Beer A). Therefore, considering the significant contrast in the ratings between Beers $\mathrm{A}$ and $\mathrm{D}$, the hypothesis that people tend to associate bitterness with lower pitch ranges and sweetness with higher pitch ranges is supported [16,17]. The general taste and flavor differences between Beers $\mathrm{A}$ and $\mathrm{D}$ were most likely the easiest to define for naïve participants. In particular, Beer A was bitter, light, and dry, whereas Beer D was, comparatively speaking, much sweeter. These results may be useful in discussing the fact that crossmodal correspondences may typically rely on explicit contrast for their effectiveness [22]. Specifically, clear differences in taste attributes (such as bitterness versus sweetness) can lead to clear differences in the pitch of sound that is matched to those taste attributes [23].

Incidentally, the resultant pitches of Beers A, B, and C (in Experiment 1) and Beer A (in Experiment 2) were contained in the range of the bitter soundtrack proposed by Reinoso Carvalho and his colleagues in [8] (Link to access the soundtrack [24]). This soundtrack has most of its sound power distributed between 50 and $350 \mathrm{~Hz}$. Intriguingly, playing this 'bitter' soundtrack enhanced the perceived alcohol content of a Belgian bitter beer.

The pitch range available for selection was different in the two experiments reported here. The ratings corresponding to Beer A appeared to be remarkably stable across both experiments (with the mean varying from $213 \mathrm{~Hz}$ to $214 \mathrm{~Hz}$ ), whereas the mean of Beer C (which was also present in both experiments) varied from $250 \mathrm{~Hz}$ to $400 \mathrm{~Hz}$. Note that it has previously been reported that 
pitch-matching tasks can depend on the set of frequencies in which those sounds are presented, making choices of pitch somewhat relative to the bandwidth that is available [25]. Even though the means of the pitch of Beer A were quite similar during both experiments, we believe that in this exercise, the participants were most likely comparing the beers and their corresponding pitch in a simpler way, rather than assigning an absolute fixed frequency to each beer. For instance, their associating tasks were perhaps assigning "low", "medium", and "high" values to their comparisons [22]. Such behavioral tasks may be related to the range frequency theory, proposed by Parducci [26], which argues that a judgment range can be psychologically sub-divided into relative sub-ranges. With the qualitative assessment that we included as part of Experiment 2, most of the participants associated bitterness with lower pitch ranges when compared with sweetness, which goes in line with part of the aforementioned theory [16,17]. However, strength also appeared to be an aspect in these comments, with some participants having reported that the stronger the beer, the higher the pitch that should be associated with it ${ }^{4}$. Interestingly, this is indeed similar to the results reported by Holt-Hansen [14], where the participants matched the lager beer with the higher alcohol content to a sound of a significantly higher pitch. This would perhaps explain why Beer $C$ was found to be statistically within the same pitch ranges as beers $A$ and D (this is visible by the high SD associated with its ratings, especially in Experiment 2). Beer $C^{\prime}$ s pitch was mostly rated towards the lower limit of the frequency range available in Experiment 2, since most of the associations were likely being driven by its bitterness. Still, since Beer $C$ is by far the strongest among them, and this might have caused its corresponding mean to be drawn, in parallel, towards the upper limit of the frequency range available in Experiment $2(50-1500 \mathrm{~Hz})$; that is, some of the participants were using relative differences in strength to define their beer-pitch associations. In other words, the pitch associated with Beer $C$ was being associated with its high bitterness by most participants (deriving in lower pitch matches) but, at the same time, it was being associated with its high strength by some participants (deriving in higher pitch matches), and this may have caused the resultant higher spreading of its ratings.

Another important observation in Experiment 2 was the fact that Beer A was rated as significantly stronger than Beer D, when actually Beer A has 4.5\% alcohol strength, and Beer D 5.1\% (see Figure 2). These results may argue with the finding that people are generally poor at estimating alcohol strength in drinks [8,27]. It seems that people tend to associate alcohol strength with flavor intensity (in this case a more bitter beer is perceived as stronger when compared to a much sweeter one), which would be another reason why these beer-pitch matching tasks seem to be more robust when relying on differences in taste attributes, rather than differences in alcohol strength.

In summary, all three of the beers present in Experiment 1 were Belgian blond bitter types. They are, indeed, quite different and with distinguishable flavors (especially when it comes to their strength). However, this sound-flavor matching process was mostly driven by the contrast of perceived bitterness versus sweetness ${ }^{5}$, and, to a lesser degree, by their differences in alcohol content (cf. [8]).

A few of the limitations of the present study are worth highlighting. For instance, the graphic interface of the frequency tone generator used in the present study was always loaded with the same starting frequency $(440 \mathrm{~Hz})$, which presents a possible source for anchoring effects [28]. In future studies, it would therefore be worthwhile to have the frequency starting point set randomly (or, perhaps, a scale that does not have a starting frequency at all), in order to eliminate any potential anchoring effects, should they exist in this situation.

In general, further studies are needed in order to uncover the basis on which such beer-pitch matches are made, and the perceptual effects of matching/non-matching tones on the drinking experience. For example, Holt-Hansen [4] reported some interesting sensations described by the participants when they heard frequencies that were not the ones chosen by them as the ideal match

4 Three participants reported stronger beers as higher in pitch.

5 This is further validated by the fact that a similar pitch-matching result from Rudmin and Cappelli's [15] study came from a colorless and odorless bitter solution made of caffeine powder and water. 
(think of watery, strong and bitter; see [29] for a review). In both of Holt-Hansen's [4,14] studies, the beer apparently tasted better at the "pitch of harmony" than when evaluated in silence. Moreover, future research could assess whether this type of multisensory matching task could be controlled by simply reorienting the participant's attention towards the attribute that is being manipulated (e.g., variations in alcohol strength versus differences in different taste attributes).

Nevertheless, it would be interesting, in future studies, to continue researching effective ways of better disentangling taste attributes and alcohol as factors. When it comes to sound-flavor associations, and as mentioned before, people commonly tend to associate alcohol strength with flavor intensity and/or flavor contrast (cf. [8,27]). Perhaps a future similar experiment could compare, for example, different-flavored alcohol-carbonated drinks with the same alcohol level (i.e., a very sweet drink versus a very bitter beer, and so on). Actually, carbonation (including its sound) can also be an important factor to be taken into consideration during future experiments. As a matter of fact, recent research has been expanding the flavor wheel in order to place mouthfeel characteristics (including carbonation) on an equal level with aroma and taste characteristics [18,19]. In our case, different levels and types of carbonation lead to different sounds, and this may have a significant effect during beer-pitch matching tasks ([30]; see [31] for an overview on the influence that the sound of carbonation can have on beverage perception).

Similar future assessments could also count on test-retest techniques for a better understanding of the liability of these types of experimental designs, especially considering the large standard deviation that is being reported here. Another interesting point to be considered in future similar assessments is the fact that higher frequency tones (when presented as single pure-tones) tend to be generally perceived as unpleasant because of their apparent loudness ${ }^{6}$, which may also be an important factor while rating 7 . Therefore, future experiments could rely on embedded loudness correction curves as part of the pitch tone generator (see [32] for an overview on the measurement of loudness). This may allow the participants to position their pitch ratings at higher ranges, with the possible mitigation of pleasure as a confounding factor.

We believe that the use of sound as a sensory enhancement in multisensory tasting experiences could provide new ways of rethinking the design and consumption of food and drinks. For example, a bitter beer accompanied by high-pitched sounds/music (such as a high-pitched piano melody) may be perceived as less bitter, making its consumption more pleasant-and potentially with less added sugar-for those who prefer sweeter beers ${ }^{8}$. This approach could be considered as complimentary to others, such as that reported by Deroy and Valentin in [34]. The analysis in the latter study used beers as taste stimuli, and revealed that there is a significant correspondence between sweetness, voluminousness, and roundness; and between bitterness, thinness, and angular shapes. Moreover, flavor-pitch associations may end up having an impact on the way people choose food and drinks, since it has been previously argued that people tend to associate different types of foods with specific types of music and soundscapes [9]. In other words, there is a whole range of crossmodal correspondences and multisensory experiential designs to be explored with beers. Actually, exploring our senses and the way in which they interact (not to mention the surprising connections between them) while we experience a food or beverage is certainly aligned with the interests of the younger generation, who are constantly looking for new ways to "promote their senses" [35]. As a matter of fact, some brewing companies (such as Inbev and The Brussels Beer Project) are already relying on similar multisensory beer experiences as a potential commercial strategy [36,37].

6 Note that in Wang et al.'s study [17], the participants rated the pleasantness of 18 single piano notes across six octaves, and the higher pitch notes were generally reported to be more pleasant. Although, in this case, it should be noted that they were piano notes, and not single pure-tones.

7 During the qualitative assessment in Experiment 2, we were able to see a few comments mentioning the fact that the frequencies were chosen based on the pleasantness induced by the sound while being played.

8 It is important to note that not everybody agrees with these propositions, and nor with their potential transcendent effect [33]. 
Acknowledgments: FRC was partly funded by the CAPES Foundation, Brazil (BEX 3488/13-6). RvE was supported by the Flemish Methusalem program (METH/14/02 to J. Wagemans), the EU Horizon 2020 program (HealthPac to J. van Opstal), and the Flemish Organization for Scientific Research (FWO). This research was also supported by the Rethinking the Senses grant from the AHRC (UK) awarded to Charles Spence (AH/L007053/1). We would also like to thank François Nelissen, Yvan de Baets, Peter Pasoli, Ilja Van Braeckel, Matthias Carlier, Maite Bezunartea, Ta Kim Hue, Marie-Paule Uwase, and Bruno da Silva for their contribution to various parts of this project. Finally, we would also like to thank the crew from 'La Brasserie de la Senne', for producing and donating the beer samples used in this study.

Author Contributions: Felipe Reinoso Carvalho is the main author of this manuscript, and led in all steps involved in this research. Qian (Janice) Wang also participated actively in all steps involved in this research. Brecht De Causmaecker worked on the characterization and classification of the beers (taste stimuli). Kris Steenhaut and Raymond Van Ee worked during the experimental design and revision process. Charles Spence was the main supervisor and editor.

Conflicts of Interest: The authors declare no conflict of interest.

\section{References}

1. Yan, K.S.; Dando, R. A crossmodal role for audition in taste perception. J. Exp. Psychol. Hum. Percept. Perform. 2015, 41, 590-596. [CrossRef] [PubMed]

2. Spence, C. Noise and its impact on the perception of food and drink. Flavour 2014, 3, 9.

3. Spence, C.; Michel, C.; Smith, B. Airplane noise and the taste of umami. Flavour 2014, 3, 2. [CrossRef]

4. Holt-Hansen, K. Extraordinary experiences during cross-modal perception. Percept. Motor Skills 1976, 43, 1023-1027. [CrossRef] [PubMed]

5. Reinoso Carvalho, F.; van Ee, R.; Touhafi, A.; Steenhaut, K.; Leman, M.; Rychtarikova, M. Assessing Multisensory Tasting Experiences by Means of Customized Sonic Cues. In Proceedings of the Euronoise 2015, Maastricht, The Netherlands, 1-3 June 2015; Volume 352, pp. 1-6.

6. Reinoso Carvalho, F.; van Ee, R.; Rychtarikova, M.; Touhafi, A.; Steenhaut, K.; Persoone, D.; Spence, C.; Leman, M. Does music influence the multisensory tasting experience? J. Sens. Stud. 2015, 30, 404-412. [CrossRef]

7. Reinoso Carvalho, F.; van Ee, R.; Rychtarikova, M.; Touhafi, A.; Steenhaut, K.; Persoone, D.; Spence, C. Using sound-taste correspondences to enhance the subjective value of tasting experiences. Front. Psychol. 2015, 6, 1309. [CrossRef] [PubMed]

8. Reinoso Carvalho, F.; Wang, Q.J.; van Ee, R.; Spence, C. The influence of soundscapes on the perception and evaluation of beers. Food Qual. Prefer. 2016, 52, 32-41. [CrossRef]

9. Reinoso Carvalho, F.; van Ee, R.; Rychtarikova, M. Matching Soundscapes and Music with Food Types. In Proceedings of the Euroregio 2016, Porto, Portugal, 13-15 June 2016; pp. 178-186.

10. Crisinel, A.-S.; Spence, C. The impact of pleasantness ratings on crossmodal associations between food samples and musical notes. Food Qual. Prefer. 2012, 24, 136-140. [CrossRef]

11. Knöferle, K.; Spence, C. Crossmodal correspondences between sounds and tastes. Psychon. Bull. Rev. 2012, 19, 992-1006. [CrossRef] [PubMed]

12. Spence, C. Eating with our ears: Assessing the importance of the sounds of consumption to our perception and enjoyment of multisensory flavour experiences. Flavour 2015, 4, 3. [CrossRef]

13. Spence, C. Multisensory flavor perception. Cell 2015, 161, 24-35. [CrossRef] [PubMed]

14. Holt-Hansen, K. Taste and pitch. Percept. Motor Skills 1968, 27, 59-68. [CrossRef] [PubMed]

15. Rudmin, F.; Cappelli, M. Tone-taste synesthesia: A replication. Percept. Motor Skills 1983, 56, 118. [CrossRef] [PubMed]

16. Crisinel, A.-S.; Spence, C. As bitter as a trombone: Synesthetic correspondences in non-synesthetes between tastes and flavors and musical instruments and notes. Atten. Percept. Psychophys. 2010, 72, 1994-2002. [CrossRef] [PubMed]

17. Wang, Q.J.; Wang, S.; Spence, C. "Turn up the taste": Assessing the role of taste intensity and emotion in mediating crossmodal correspondences between basic tastes and pitch. Chem. Sens. 2016, 41, 345-356. [CrossRef] [PubMed]

18. Lewis, M.J.; Young, T.W. Brewing; Springer Science \& Business Media: New York, NY, USA, 2012.

19. Langstaff, S.A.; Guinard, J.X.; Lewis, M.J. Sensory evaluation of the mouthfeel of beer. J. Am. Soc. Brew. Chem. 1991, 49, 54-59. 
20. Ale Beer Styles. Available online: http://www.beermonthclub.com/beer-style-guide-ales.htm (accessed on 23 October 2016).

21. Field, A. Discovering Statistics Using IBM SPSS; Sage: Los Angeles, CA, USA, 2013.

22. Gallace, A.; Spence, C. Multisensory synesthetic interactions in the speeded classification of visual size. Percept. Psychophys. 2006, 68, 1191-1203. [CrossRef] [PubMed]

23. Spence, C. Crossmodal correspondences: A tutorial review. Atten. Percept. Psychophys. 2011, 73, 971-995. [CrossRef] [PubMed]

24. Sonic Seasoning with Belgian Beers. Available online: http://sonicseasoningbeer.tumblr.com/ (accessed on 25 October 2016).

25. Pedley, P.E.; Harper, R.S. Pitch and the vertical localization of sound. Am. J. Psychol. 1959, 72, 447-449. [CrossRef]

26. Parducci, A. Category judgment: A range-frequency model. Psychol. Rev. 1965, 72, 407-418. [CrossRef] [PubMed]

27. Stafford, L.D.; Fernandes, M.; Agobiani, E. Effects of noise and distraction on alcohol perception. Food Qual. Prefer. 2012, 24, 218-224. [CrossRef]

28. Stewart, N. The cost of anchoring on credit-card minimum repayments. Psychol. Sci. 2009, $20,39-41$. [CrossRef] [PubMed]

29. Spence, C.; Wang, Q. Wine \& music (III): So what if music influences taste? Flavour 2015, 4, 36.

30. Zampini, M.; Spence, C. Modifying the multisensory perception of a carbonated beverage using auditory cues. Food Qual. Prefer. 2005, 16, 632-641. [CrossRef]

31. Spence, C.; Wang, Q. Sensory expectations elicited by the sounds of opening the packaging and pouring a beverage. Flavour 2015, 4, 35. [CrossRef]

32. Stevens, S.S. The measurement of loudness. J. Acoust. Soc. Am. 1955, 27, 815-829. [CrossRef]

33. Hunt, A. Can Music Improve Wine? JanciaRobinson.com. Published on 5 May. Available online: http: //www.jancisrobinson.com/articles/can-music-improve-wine (accessed on 25 October 2016).

34. Deroy, O.; Valentin, D. Tasting liquid shapes: Investigating the sensory basis of cross-modal correspondences. Chemosens. Percept. 2011, 4, 80-90. [CrossRef]

35. Spence, C.; Piqueras-Fiszman, B. The Perfect Meal: The Multisensory Science of Food and Dining; Wiley-Blackwell: Oxford, UK, 2014.

36. Birkner, C. Stella Artois and the Roots Created a Music Video You Can Taste-Sounds Enhance the Beer's Sweet and Bitter Notes. Add Week Magazine, Advertising \& Branding. Published on 19 August. Available online: http:/ / www.adweek.com/news/advertising-branding/stella-artois-and-roots-createdmusic-video-you-can-taste-173057 (accessed on 20 October 2016).

37. Reinoso Carvalho, F.; Velasco, C.; van Ee, R.; Leboeuf, Y.; Spence, C. Music influences hedonic and taste ratings in beer. Front. Psychol. 2016, 7, 636. [CrossRef] [PubMed]

(C) 2016 by the authors; licensee MDPI, Basel, Switzerland. This article is an open access article distributed under the terms and conditions of the Creative Commons Attribution (CC-BY) license (http://creativecommons.org/licenses/by/4.0/). 